\title{
The evolving role of CT and MRI in atherothrombotic evaluation and management
}

\author{
Valentin Fuster
}

Morbid cardiovascular events often happen suddenly and with little warning. Risk assessment scores based on conventional risk factors are not, however, uniformly predictive and might not capture other genetic, familial or less-welldefined risk factors. In addition, a large proportion of patients classified as being at intermediate or high risk ( $40 \%$ and $25 \%$ of the US population, respectively) might never experience an event. This disparity is especially true of those in the intermediate group. The reference standard for the evaluation of coronary artery disease (CAD) is angiographic visualization and quantification of significant coronary stenoses. The invasive nature of this test and related costs and logistic issues, however, limit its potential use in large populations. Thus, the development of widely applicable, more-accurate, early detection and stratification strategies for cardiovascular disease risk is a major public-health requirement.

Although notable research efforts are focused on the identification of serum cardiovascular disease markers, whether such surrogate approaches will have enough sensitivity to reflect consistently the atherothrombotic process in the vessel wall remains unclear. Thus, another important route of study is the role of advancing noninvasive imaging technologies.

Of the CT-based approaches, electron beam CT and, to a much greater extent, multidetectorrow CT have emerged as robust methods, both of which can evaluate coronary artery calcium without contrast enhancement, and detect coronary luminal narrowing in iodinated-contrastenhanced angiography studies. Calcium scoring, for which electron beam CT is currently the gold standard, enables estimation of future coronary stenosis, offers prognostic information additional to that provided by traditional risk factor assessment, and might prove useful in the assessment of therapeutic outcomes.

Contrast-enhanced angiography, for which multidetector-row CT is generally the preferred method, enables highly accurate assessment of significant coronary luminal narrowing and ...magnetic

resonance

and CT

technologies

are likely to

gain wider

acceptance

by the medical

community

and become

more available

for use in large

populations...

$V$ Fuster is the Editorin-Chief of Nature

Clinical Practice

Cardiovascular

Medicine.

Competing interests

The author declared he has no competing interests.

www.nature.com/clinicalpractice doi:10.1038/ncpcardio0249 might help to tailor clinical decisions for individual patients. Patency of bypass grafts can be reliably assessed and results for in-stent restenosis seem good. Plaque characterization might also be possible. With rapid evolution in CT technology, the development of 64-slice scanners and high-resolution images, coronary CT will contribute greatly to understanding, diagnosis and management of coronary atherothrombotic disease.

Cardiovascular MRI encompasses various different techniques to enable comprehensive evaluation of the burden of disease, and to aid diagnosis and systemic arterial therapy. The Framingham Risk Score for CAD was shown in asymptomatic individuals to be associated with noninvasive aortic plaque burden on MRI. Our research group has demonstrated that noninvasive carotid and aortic plaque burden on $\mathrm{MRI}$ is more significantly associated with presence or absence of CAD than the Framingham Risk Score. In addition, high-resolution MRI (i.e. black-blood and high-speed imaging and detection coils) is a potential leader in noninvasive imaging methods for plaque characterization.

Coronary MRI is technically challenging, and spatial coverage, temporal resolution and image quality are currently limited. Two emerging MRI technologies will, however, prove extremely useful over the next 3-5 years. The first, global coherent free precession, is a new method that can depict vascular anatomy and vascular function (blood flow) simultaneously without the need for contrast agents or ionizing radiation. The second, high-resolution contrast-enhanced molecular MRI, enables localized coronary plaque characterization through the peripheral injection of contrast agents targeted to specific cells, molecules or processes, such as extra cellular lipid, macrophages and thrombi. With such advances, magnetic resonance and CT technologies are likely to gain wider acceptance by the medical community and become more available for use in large populations for these purposes. 